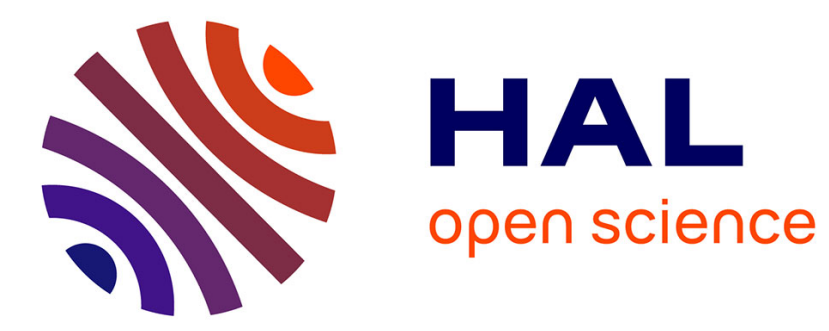

\title{
Optimal estimation of diffusion in DW-MRI by high-order MRF-based joint deformable registration and diffusion modeling
}

Evgenios N. Kornaropoulos, Evangelia I. Zacharaki, Pierre Zerbib, Chieh Lin, Alain Rahmouni, Nikos Paragios

\section{To cite this version:}

Evgenios N. Kornaropoulos, Evangelia I. Zacharaki, Pierre Zerbib, Chieh Lin, Alain Rahmouni, et al.. Optimal estimation of diffusion in DW-MRI by high-order MRF-based joint deformable registration and diffusion modeling. WBIR 2016 - 7th International Workshop on Biomedical Image Registration, Jul 2016, Las Vegas, Nevada, United States. hal-01324229

\section{HAL Id: hal-01324229 \\ https://hal.science/hal-01324229}

Submitted on 31 May 2016

HAL is a multi-disciplinary open access archive for the deposit and dissemination of scientific research documents, whether they are published or not. The documents may come from teaching and research institutions in France or abroad, or from public or private research centers.
L'archive ouverte pluridisciplinaire HAL, est destinée au dépôt et à la diffusion de documents scientifiques de niveau recherche, publiés ou non, émanant des établissements d'enseignement et de recherche français ou étrangers, des laboratoires publics ou privés. 


\section{Optimal estimation of diffusion in DW-MRI by high-order MRF-based joint deformable registration and diffusion modeling}

\author{
Evgenios N. Kornaropoulos ${ }^{1}$ \\ evgenios.kornaropoulosecp. fr \\ Chieh $\operatorname{Lin}^{3}$ \\ sophieclinegmail.com
}

\author{
Evangelia I. Zacharaki ${ }^{1}$ \\ evangelia.zacharaki@ecp.fr
}

Alain Rahmouni ${ }^{2}$

alain.rahmounieaphp.fr

\author{
Pierre Zerbib ${ }^{2}$ \\ pierre.zerbibeaphp.fr \\ Nikos Paragios ${ }^{1}$ \\ nikos.paragiosdecp.fr
}

${ }^{1}$ Center for Visual Computing, CentraleSupélec, Inria, Université Paris-Saclay, France

${ }^{2}$ Centre Hospitalier Universitaire Henri-Mondor, France

${ }^{3}$ Chang Gung Memorial Hospital, Taiwan

\begin{abstract}
Over the last years, the apparent diffusion coefficient $(A D C)$, computed from diffusion-weighted magnetic resonance $(D W-M R)$ images, has become an important imaging biomarker for evaluating and managing patients with neoplastic or cerebrovascular disease. Standard methods for the calculation of ADC ignore the presence of noise and motion between successive (in time) DW-MR images acquired by changing the b-value. In order to accurately quantify the diffusion process during image acquisition, we introduce a method based on a high-order Markov Random Field (MRF) formulation that jointly registers the $D W$ MR images and models the spatiotemporal diffusion. Spatial smoothness on the ADC map, as well as spatiotemporal deformation smoothness, is imposed towards producing anatomically meaningful representations. The high-order dependencies in our MRF model are handled through Dual Decomposition. Performance of registration is compared to a state-of-the art registration approach in terms of obtained fitting error of the diffusion model in the core of the tumor. Preliminary results reveal a marginally better performance of our method when compared against the standard ADC map used in clinical practice, which indicates its potential as a means for extracting imaging biomarkers.
\end{abstract}

\section{Introduction}

Since the proposal of background body signal suppression [25], DW-MRI has gained clinical impetus for the detection of primary tumors and metastases throughout the whole body [10], [17]. Besides the qualitative anatomical information [3], [1], [27], [22], quantitative analysis of whole-body DW-MRI is becoming possible by scanning the patient with different $b$-values (a parameter determining the strength and duration of the diffusion gradients). The apparent diffusion coefficient, which reflects the gradient of water diffusivity in the body, can be subsequently computed at each voxel and displayed as a parametric map (ADC map) [14]. ADC has been examined not only for the characterization and staging of lesions but also for the response to treatment [16], [8], [28], [15]. The calculation of ADC was performed without motion or artifact correction in the previous studies. It is reported though that ADC is a parameter susceptible to artifacts, the most frequent of all being patient's motion and breathing, resulting in misregistration of the images obtained with different b-values [1], [13]. Therefore, in order to correctly visualize diffusion in biological tissues, image registration should precede the ADC calculation [10]. Specifically, due to non-linear motion (cardiac pulses, respiration), non-rigid alignment of the DW-MRI sequence becomes necessary [23].

In this paper, we propose a joint deformable registration and appearance model computation framework. The deformation model maps each one of the $m$ DW images in a common space (the ADC space), whereas the appearance model describes the signal intensity related to the diffusion of water molecules that takes place during DW-MRI acquisition. The unknown variables of the combined deformation and appearance model correspond to a $m+2$-layer graphical model, $m$ layers that represent the $m$ 3D deformation fields, one for each DW image, and two layers for the two parameters of the appearance model, namely the 3D ADC map and the 3D signal intensity without diffusion weighting (explained later with more details). The latent deformation variables are obtained using the discrete formulation introduced in [7]. The $m+2$ layers are interconnected in order to achieve consistency between the predicted diffusion 
(by the appearance model) and the diffusion calculated by the registered DW images. Each deformation field depends thus on the 2-layers appearance model, which amounts to a high-order dependency that requires a high-order inference scheme. Spatial smoothness constraints on the parameters of the appearance model and the deformations are imposed through pairwise interactions. Moreover, the $m$ layers deformation fields have pairwise interconnections (based on proximity of b-values) towards imposing smoothness on the change of deformation through time (the images sequence is acquired with increasing b-values).

Our approach lies between frameworks of concurrent segmentation and registration [29], [30], [18], [2] and image reconstruction and modeling [21], [19]. The main difference of our approach with respect to the concurrent segmentation and registration works is that, our ADC variables are continuous and have unique values on a voxel-wise basis, whereas segmentation labels are discrete and common within regions. In that sense, methods for image reconstruction [21] and denoising [20] are closer to our approach; those however are defined in a single spatial domain and do not require deformable image registration. On the other hand, methods for recovering appearance change and motion in image sequences target a similar goal, but they account usually for global motion or smooth deformations, as well as systematic changes in appearance exhibiting spatial or temporal structure that can be modeled [5]. Intensity changes in domain-specific cases are captured by learning models using training examples. Such training images are not available in our case where the tumor appears in different areas of the body.

By elaborating more on concurrent segmentation and registration frameworks, we aim at finding the optimal deformation fields of the DW-MR images together with the optimal ADC values in a one-shot optimization approach. Alchatzidis et al. [2] proposed a joint group-wise registration and segmentation method for brain atlas selection, but they optimized the deformation fields iteratively, whereas Sotiras et al. [24] didn't include a segmentation layer in their group-wise, one-shot registration method. In our case the one-shot optimization problem imposes additional challenges. To this end, Dual Decomposition (DD) for MRF optimization [11] is used to determine the optimal solution to the joint problem, in which linear programming and duality [12] are incorporated. It is also worth to mention that our framework is modular with respect to the registration/annotation grid and can be adapted to the application domain. Finally, to the best of our knowledge, we are the first to propose a DW-MRI registration scheme that optimizes explicitly the derived ADC map. Very recently, two other works of DW-MRI registration were published [9], [26] aiming at computing the ADC without motion artifacts. Guyader at al. [9] examined the influence of pairwise regis- tration on the ADC calculation, while Veeraraghavan et al. [26] proposed a group-wise DW-MRI registration method that requires structure segmentation, thus is not completely automated. Both methods (i) use mutual information criterion for image similarity which is less appropriate than the fitting error to a diffusion model we are using. (ii) calculate the motion for each image independently without considering temporal consistency (smooth transition in time) and (iii) extract the ADC by voxel-wise curve fitting from the warped images without accounting for spatial constraints.

The remainder of this paper is organized as follows: section 2 describes the combined registration and ADC calculation strategy, while experimental validation is presented in the following section. Discussion and future directions conclude the paper.

\section{Graph-based joint DW image registration and diffusion modelling}

Let us consider a sequence of $m$ DW-MR images, each one described by intensity values $s_{t}(x), t=1, \ldots, m$, with $x \in \Omega_{t}, \Omega_{t} \in \mathbf{R}^{\mathbf{3}}$. On top of that, let's consider an extra image $z(x)$ with $x \in \Omega_{z}$ which represents the ADC template corresponding to the previous image sequence. This ADC template is regarded as the reference frame of an optimal alignment among the DW-MR images. We therefore seek for two results: a set of deformations $d=\left\{d: x_{R}=\right.$ $\left.d_{t}\left(x_{t}\right), t=\{1, \ldots, m\}\right\}$ which map mutually corresponding points from the $m$-image spaces to the same point of the reference frame $\Omega_{z}$ and the optimal - in terms of spatial consistency and smoothness - ADC values that can be calculated when all $m$-images are mutually aligned. This joint mapping and ADC refinement aims at (i) correcting deformations due to (local) organ deformations or motion, (ii) ensure temporal consistency in the diffusion process and (iii) impose spatial consistency on the deformation fields and the derived ADC map. The appearance model used for the calculation of the ADC, as well as the deformation model, are jointly optimized to define the reference pose. In the following section, the problem formulation is presented, first for the case of negligible deformations and then for all possible deformations.

\subsection{Appearance (diffusion) model and MAP esti- mation}

In DWI, we assume that the intensity values of the images depend mainly on the amount of signal loss occurred during the application of two equally rectangular gradients before and after the $180^{\circ}$-refocusing pulse [4]. The relation between the signal attenuation expressed by the signal intensity $s$ and the diffusion of the water molecules in a microscopic level is known to be exponential [14]. Having acquired the images with high $\mathrm{b}$ values $\left(>50 \mathrm{~s} / \mathrm{mm}^{2}\right)$, we can assume that the DW images are insensitive to vascular 
capillary perfusion and diffusion is the only type of motion present, thus the ADC is equal to the water diffusion [10]. Therefore the relation between the ADC (referred to with the letter $z$ ) and the signal intensity $s$ can be modeled as:

$$
\mathbf{s}_{\mathbf{t}}=\mathbf{s}_{\mathbf{0}} \mathbf{e}^{-\mathbf{b}_{\mathbf{t}} \cdot \mathbf{z}}
$$

where $\mathbf{s}_{\mathbf{t}}$ and $b_{t}$ the image intensity vector and b-value at instant $t$ respectively, $\mathbf{s}_{\mathbf{0}}$ the signal attenuation for $b=0$ and $\mathbf{z}$ is the ADC vector (all vectors are linearized 3D matrices). Denoting with $\mathbf{y}_{\mathbf{t}}$ the natural logarithm of the image vector $\mathbf{s}_{\mathbf{t}}$, the relation between the image vector and the ADC can be expressed as:

$$
\mathbf{y}_{\mathbf{t}}=-\mathbf{b}_{\mathbf{t}} \mathbf{z}+\mathbf{y}_{\mathbf{0}}+\mathbf{n}_{\mathbf{t}}
$$

where $\mathbf{n}_{\mathbf{t}}$ the noise vector. We are assuming noise to be zero mean i.i.d., thus the multivariate $\operatorname{pdf}$ of $\mathbf{n}_{\mathbf{t}}$ is given by:

$$
P\left(\mathbf{n}_{\mathbf{t}}\right)=\frac{\mathbf{1}}{(\mathbf{2} \pi)^{\frac{\mathrm{M}}{2}} \sigma_{\mathbf{n}}^{\mathrm{M}}} \exp \left\{-\frac{\mathbf{1}}{\mathbf{2} \sigma_{\mathbf{n}}^{\mathbf{2}}} \mathbf{n}_{\mathbf{t}}^{\mathbf{T}} \mathbf{n}_{\mathbf{t}}\right\}
$$

where $M$ the size of the linearized noise vector and $\sigma_{n}^{2}$ denotes the variance of the noise process. If all $m$ DW-MR images are perfectly aligned, the standard way of estimating ADC is by calculating the slope of a line fitted on the plot of the logaritm of the relative signal intensities of the tissue against the b-values:

$$
\mathbf{z}=\frac{\sum_{\mathbf{i}=\mathbf{1}}^{\mathbf{m}}\left(\mathbf{b}_{\mathbf{i}}-\overline{\mathbf{b}}\right)\left(\left(\mathbf{y}_{\mathbf{i}}-\overline{\mathbf{y}}\right)\right.}{\sum_{\mathbf{i}=\mathbf{1}}^{\mathbf{m}}\left(\mathbf{b}_{\mathbf{i}}-\overline{\mathbf{b}}\right)^{2}}
$$

where $\bar{b}, \overline{\mathbf{y}}$ the mean b-value and mean of the natural logarithm of the image vector respectively. We approach the calculation of $\mathbf{z}$, as well as $\mathbf{y}_{\mathbf{0}}$ using the Maximum a Posteriori (MAP) technique:

$$
\hat{\mathbf{z}}, \hat{\mathbf{y}_{\mathbf{0}}}=\underset{\mathbf{z}, \mathbf{y}_{\mathbf{0}}}{\operatorname{argmax}} \mathbf{P}\left(\mathbf{z}, \mathbf{y}_{\mathbf{0}} \mid \mathbf{y}_{\mathbf{1}}, \cdots, \mathbf{y}_{\mathbf{m}}\right)
$$

The random fields $\mathbf{z}$ and $\mathbf{y}_{\mathbf{0}}$ are assumed to be statistically independent in this study, as they refer to two independent processes. From Bayes' rule, equation (4) can be written as:

$$
\hat{\mathbf{z}}, \hat{\mathbf{y}_{\mathbf{0}}}=\underset{\mathbf{z}}{\operatorname{argmax}} \frac{\mathbf{P}\left(\mathbf{y}_{\mathbf{1}}, \cdots, \mathbf{y}_{\mathbf{m}} \mid \mathbf{z}, \mathbf{y}_{\mathbf{0}}\right) \mathbf{P}(\mathbf{z}) \mathbf{P}\left(\mathbf{y}_{\mathbf{0}}\right)}{\mathbf{P}\left(\mathbf{y}_{\mathbf{1}}, \cdots, \mathbf{y}_{\mathbf{m}}\right)}
$$

Since the denominator is not a function of $\mathbf{z}$ nor of $\mathbf{y}_{\mathbf{0}}$, it does not influence their estimation and therefore can be ignored. Taking the log probability of posterior probability:

$$
\begin{aligned}
& \hat{\mathbf{z}}, \hat{\mathbf{y}_{\mathbf{0}}}=\underset{\mathbf{z}, \mathbf{y}_{\mathbf{0}}}{\operatorname{argmax}}\left\{\left[\log \mathbf{P}\left(\mathbf{y}_{\mathbf{1}}, \cdots, \mathbf{y}_{\mathbf{m}} \mid \mathbf{z}, \mathbf{y}_{\mathbf{0}}\right)\right.\right. \\
& \left.\left.+\log \mathbf{P}(\mathbf{z})+\log \mathbf{P}\left(\mathbf{y}_{\mathbf{0}}\right)\right]\right\}
\end{aligned}
$$

Next we solve equation (7) assuming there is no deformation between the DW images.
From equation (7), since $\mathbf{n}_{\mathbf{t}}$ 's are assumed to be independent:

$$
\begin{aligned}
& \hat{\mathbf{z}}, \hat{\mathbf{y}_{\mathbf{0}}}=\underset{\mathbf{z}, \mathbf{y}_{\mathbf{0}}}{\operatorname{argmax}}\left\{\left[\log \prod_{\mathbf{t}=\mathbf{1}}^{\mathbf{m}} \mathbf{P}\left(\mathbf{y}_{\mathbf{t}} \mid \mathbf{z}, \mathbf{y}_{\mathbf{0}}\right)\right.\right. \\
& \left.\left.+\log \mathbf{P}(\mathbf{z})+\log \mathbf{P}\left(\mathbf{y}_{\mathbf{0}}\right)\right]\right\}=\underset{\mathbf{z}, \mathbf{y}_{\mathbf{0}}}{\operatorname{argmax}}\left\{\left[\sum_{t=1}^{m} \log \mathbf{P}\left(\mathbf{y}_{\mathbf{i}} \mid \mathbf{z}, \mathbf{y}_{\mathbf{0}}\right)\right.\right. \\
& \left.\left.+\log \mathbf{P}(\mathbf{z})+\log \mathbf{P}\left(\mathbf{y}_{\mathbf{0}}\right)\right]\right\}
\end{aligned}
$$

Since noise is assumed to be i.i.d. Gaussian, from equations (2) and (3) we have:

$$
\begin{aligned}
& \mathbf{P}\left(\mathbf{y}_{\mathbf{1}}, \cdots, \mathbf{y}_{\mathbf{m}} \mid \mathbf{z}, \mathbf{y}_{\mathbf{0}}\right)= \\
& {\left[\sum_{t=1}^{m} \log \frac{1}{\left(2 \pi \sigma_{n}^{2}\right)^{\frac{M}{2}}} \exp \left\{-\frac{\left\|\mathbf{y}_{\mathbf{t}}+\mathbf{b}_{\mathbf{t}} \mathbf{z}-\mathbf{y}_{\mathbf{0}}\right\|^{\mathbf{2}}}{\mathbf{2} \sigma_{\mathbf{n}}^{\mathbf{2}}}\right\}\right]=} \\
& \sum_{t=1}^{m} \frac{-\left\|\mathbf{y}_{\mathbf{t}}+\mathbf{b}_{\mathbf{t}} \mathbf{z}-\mathbf{y}_{\mathbf{0}}\right\|^{\mathbf{2}}}{2 \sigma_{n}^{2}}-\frac{M}{3} \log \left(2 \pi \sigma_{n}^{2}\right)
\end{aligned}
$$

Substituting equation (9) in equation (8) we obtain:

$$
\begin{aligned}
& \hat{\mathbf{z}}, \hat{\mathbf{y}_{\mathbf{0}}}= \\
& \underset{\mathbf{z}, \mathbf{y}_{\mathbf{0}}}{\operatorname{argmax}}\left\{\left[\sum_{t=1}^{m} \frac{-\left\|\mathbf{y}_{\mathbf{t}}+\mathbf{b}_{\mathbf{t}} \mathbf{z}-\mathbf{y}_{\mathbf{0}}\right\|^{\mathbf{2}}}{2 \sigma_{n}^{2}}\right.\right. \\
& \left.\left.+\log \mathbf{P}(\mathbf{z})+\log \mathbf{P}\left(\mathbf{y}_{\mathbf{0}}\right)\right]\right\}
\end{aligned}
$$

The derivation of a solution for ADC based on equation (10) is the first contribution of our work, in which the ADC can be calculated based on the data (DW-MR images) and some prior knowledge about context dependencies on ADC map and $\mathbf{y}_{\mathbf{0}}$ values, acting as regularization constraints.

\subsection{MAP estimation for joint appearance and deformation model}

Assuming now that some motion has occurred during the acquisition process of the DW-MR images, equation (2) takes the form:

$$
\mathbf{y}_{\mathbf{t}} \circ \mathbf{D}_{\mathbf{t}}=-\mathbf{b}_{\mathbf{t}} \mathbf{z}+\mathbf{y}_{\mathbf{0}}+\mathbf{n}_{\mathbf{t}}
$$

where $\mathbf{D}_{\mathbf{t}}$ denotes the deformation field that maps $\mathbf{s}_{\mathbf{t}}$ to the ADC template space. In this case, we would like to compute the $m$ deformation fields that would bring the $m$ DW-MR images to a common reference space in which the ADC map can be accurately estimated. Let's denote the geometric deformation that maps the DW-MR image $\mathbf{s}_{\mathbf{t}}$ to the common (ADC) space with $\mathbf{D}_{\mathbf{t}}(\mathbf{x})=\mathbf{x}+\mathbf{d}_{\mathbf{t}}(\mathbf{x})$, where $\mathbf{d}_{\mathbf{t}}$ is the displacement field of image $s_{t}$. The joint posterior probability for the appearance model parameters and the set of 
displacement fields $\left[\mathbf{d}_{\mathbf{1}}, \cdots, \mathbf{d}_{\mathbf{m}}\right]$ is given by:

$$
\begin{aligned}
& \mathbf{P}\left(\mathbf{d}_{1}, \cdots, \mathbf{d}_{\mathbf{m}}, \mathbf{z}, \mathbf{y}_{\mathbf{0}} \mid \mathbf{y}_{\mathbf{1}}, \cdots, \mathbf{y}_{\mathbf{m}}\right)= \\
& \frac{\mathbf{P}\left(\mathbf{y}_{\mathbf{1}}, \cdots, \mathbf{y}_{\mathbf{m}} \mid \mathbf{d}_{\mathbf{1}}, \cdots, \mathbf{d}_{\mathbf{m}}, \mathbf{z}, \mathbf{y}_{\mathbf{0}}\right) \mathbf{P}\left(\mathbf{d}_{\mathbf{1}}, \cdots, \mathbf{d}_{\mathbf{m}}, \mathbf{z}, \mathbf{y}_{\mathbf{0}}\right)}{\mathbf{P}\left(\mathbf{y}_{\mathbf{1}}, \cdots, \mathbf{y}_{\mathbf{m}}\right)}
\end{aligned}
$$

The random fields $\mathbf{d}_{\mathbf{t}}, \mathbf{z}$ and $\mathbf{y}_{\mathbf{0}}$ are once again assumed to be statistically independent, as they refer to three independent processes. Therefore, the joint MAP solution could be derived similarly as in the previous section, resulting in the following optimization problem:

$$
\begin{aligned}
& \hat{\mathbf{z}}, \hat{\mathbf{y}_{\mathbf{0}}}, \hat{\mathbf{d}_{\mathbf{1}}}, \cdots, \hat{\mathbf{d}_{\mathbf{m}}}= \\
& \underset{\mathbf{z}, \mathbf{y}_{\mathbf{0}}, \mathbf{d}_{\mathbf{1}}, \cdots, \mathbf{d}_{\mathbf{m}}}{\operatorname{argmax}}\left\{\left[\sum_{t=1}^{m} \frac{-\left\|\mathbf{y}_{\mathbf{t}} \circ \mathbf{D}_{\mathbf{t}}+\mathbf{b}_{\mathbf{t}} \mathbf{z}-\mathbf{y}_{\mathbf{0}}\right\|^{\mathbf{2}}}{2 \sigma_{n}^{2}}\right.\right. \\
& \left.\left.+\log \mathbf{P}(\mathbf{z})+\log \mathbf{P}\left(\mathbf{y}_{\mathbf{0}}\right)+\log \mathbf{P}\left(\mathbf{d}_{\mathbf{1}}, \cdots, \mathbf{d}_{\mathbf{m}}\right)\right]\right\}
\end{aligned}
$$

The latter equation for computing a joint solution for $\mathrm{ADC}$ and deformation fields, using some priors on context dependencies on $\mathbf{z}, \mathbf{y}_{\mathbf{0}}$ and deformation fields, is the second contribution of our work.

\subsection{MRF Formulation of Joint Problem}

We formulate our joint DW-MRI registration and ADC modeling problem using Markov Random Fields (MRF). The joint model is parameterized by a set of $m+2$ isomorphic grid graphs $G=\left\{G_{1}, \ldots, G_{m+2}\right\}$, each of the first $m$ superimposed onto the corresponding DW-MR image, whereas the last two grid graphs are superimposed onto the ADC map that we want to compute and the $\mathbf{y}_{0}$ image vector respectively. To this end, we define three sets of labels, corresponding to the three different types of variables in our model. The label set $\mathscr{L}_{d}$ is a quantized version of the displacement space. A label assignment $l_{p}^{d} \in \mathscr{L}^{d}$ (with $p \in \mathscr{V}_{d}$ ) is equivalent to displacing the control point $p$ by displacement $\mathbf{d}_{\mathbf{p}}$. Correspondingly, the labels $l_{p}^{z} \in \mathscr{L}^{z}$ and $l_{p}^{y_{0}} \in \mathscr{L}^{y_{0}}$ are quantized versions of the ADC and $\mathbf{y}_{\mathbf{0}}$ intensities, respectively.

For every control point in each grid $i$ there is a node $p$ that represents either its displacement if the grid is a deformation grid either the ADC or the $y_{0}$ value in case of the last two grids respectively ( $p \in \mathscr{V}$, with $\mathscr{V}$ denoting the set of all nodes on a grid that encode the latent variable). Regarding the deformation, it is evaluated on the deformation grid's control point $p$ as $D(x)=x+\sum_{p \in G} \eta(\|x-p\|) d_{p}$, where $d_{p}$ is the displacement of the grid's control point $p$. By moving the grid's control points, we end up deforming the DW-MR images. In the end, the displacement of a voxel $\mathrm{x}$ of the image will be determined by the control points displacements and the influence of each control point on $x$, which is given by the projection function $\eta$ (a cubic B-splines in our case). Due to the isomorphic nature of the grid, $p$ indexes a common to all grids control point position. Moreover, the nodes in the graph are connected with a set of edges $\mathscr{E}$ that encode the interactions between the deformation and the ADC variables. The first type of dependencies between the nodes is a high-order dependency term that relates to the coupling term, which penalises any deviation due to registration of the image intensity value from the attenuation value calculated based on equation (1). The second type of dependencies in our energy formulation is related to temporal constistency of the deformation fields, which means that we assume that the deformations occurring on the image query should change smoothly from one image to the other. This term is applicable only on edges connecting nodes on different deformation grids (inter-deformation dependency). The third, fourth and fifth terms relate to the smoothness constraints and are applicable only on edges connecting nodes on the same grid (intra-deformation, intra-ADC and intra-b0 dependency respectively) (see figure 1, left).

Energy terms: The constructed graph is associated with an energy, consisting of four pairwise terms:

$$
\begin{aligned}
& E_{\text {joint }}\left(d_{1}, \cdots, d_{m}, z, y_{0}\right)= \\
& \lambda_{c} \sum_{t=1}^{m} \sum_{p \in G_{t}} \sum_{p \in G_{m+1}} \sum_{p \in G_{m+2}} V_{c}\left(l_{p}^{d_{t}}, l_{p}^{z}, l_{p}^{y_{0}}\right) \\
& +\lambda_{\text {inter }} \sum_{t=1}^{m-1} \sum_{p \in G_{t}} V_{\text {inter }}\left(l_{p}^{d_{t}}, l_{p}^{d_{t+1}}\right) \\
& +\lambda_{\text {intra }} \sum_{t=1}^{m} \sum_{p \in G_{i}} \sum_{q \in\left(N(p) \cap G_{i}\right)} V_{i n t r a^{1}}\left(l_{p}^{d_{t}}, l_{q}^{d_{t}}\right) \\
& +\lambda_{\text {intra }}{ }^{2} \sum_{p \in G_{m+1}} \sum_{q \in\left(N(p) \cap G_{m+1}\right)} V_{i n t r a^{2}}\left(l_{p}^{z}, l_{q}^{z}\right) \\
& +\lambda_{\text {intra }} \sum_{p \in G_{m+2}} \sum_{q \in\left(N(p) \cap G_{m+2}\right)} V_{i n t r a^{3}}\left(l_{p}^{y_{0}}, l_{q}^{y_{0}}\right)
\end{aligned}
$$

where $V_{c}$ is the coupling potential of a third-order dependency, $V_{\text {inter }}$ is a pair-wise potential for temporal regularization, $V_{\text {intran }^{n}}(\cdot, \cdot), n=1, \cdots, 3$ are the three pairwise potentials imposing spatial regularization within each graph, and $N$ represents the spatial connectivity of the nodes. The constants $\lambda_{c}, \lambda_{\text {inter }}$ and $\lambda_{\text {intra }^{n}}, n=1, \cdots, 3$ are used to weight the different types of potentials.

Coupling term: It penalizes deformations that lead to an increase in the matching error among $z, y_{0}$ and the signal attenuation at a given instant $t$, based on equation (13). It is applicable on a third-order clique consisted by the corresponding control points $p$ (same spatial location) on the 

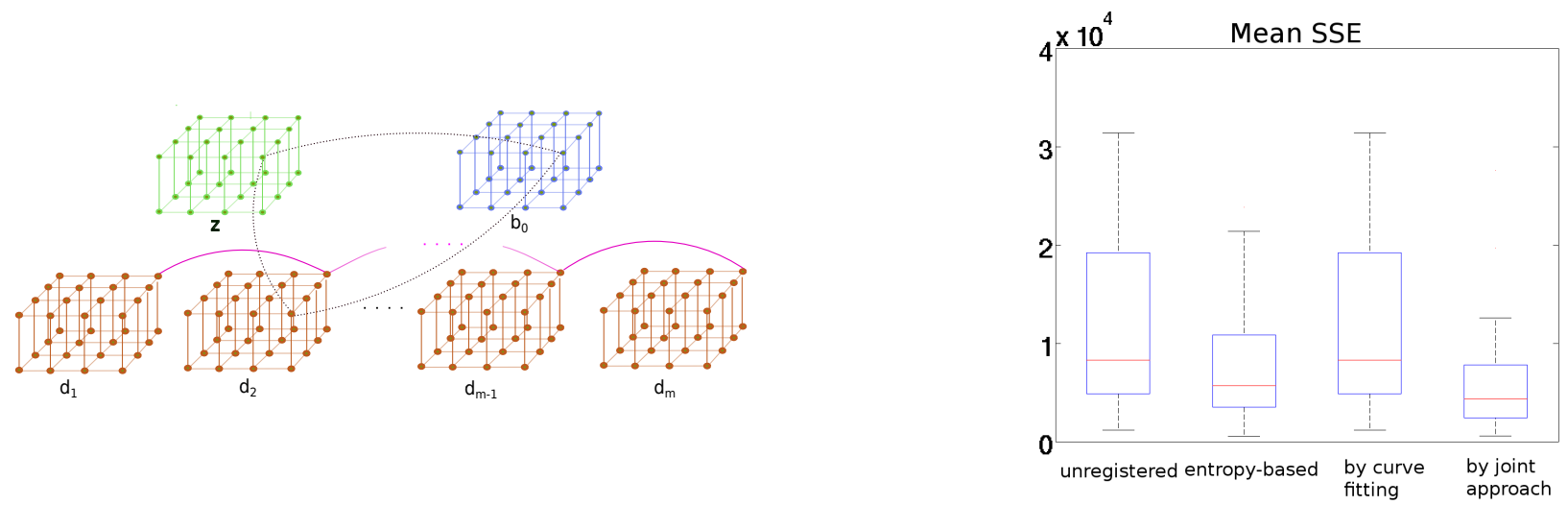

Figure 1: Left figure: The node and the edge system of all the connected graphs. With green, blue and brown color the relationship within the grid nodes belonging to ADC, $\mathbf{y}_{\mathbf{0}}$ and each of the deformation field respectively (intra connections). The black dotted edge represents the third-order dependency among deformation field and appearance model (coupling relationship), whereas pink edges denote the temporal relationship between successive deformatio fields (inter relationship). Right figure: Boxplots of the mean SSE based on ADC maps derived by the four different examined cases.

deformation, the $\mathbf{z}$ and the $\mathbf{y}_{\mathbf{0}}$ grid:

$$
\begin{aligned}
& V_{c}\left(l_{p}^{d_{t}}, l_{p}^{z}, l_{p}^{y_{0}}\right)=\int_{\Omega_{s_{t}}} \bar{\eta}\left(\left\|x_{t}-p\right\|\right) \\
& \left(y\left(x_{t}+d^{l_{p}^{d_{t}}}\right)+b_{t} z^{l_{p}^{z}}-y_{0}^{l_{p}^{y_{0}}}\right)^{2} d x_{t}
\end{aligned}
$$

This term is our data term as it connects the DW-MR images with all three types of variables. It is defined on the image domain $\Omega$ and a function $\bar{\eta}$ is used to back project the voxel-wise information on the grids' control points, by determining how much voxel $x$ influences the control point $p$.

Spatial and temporal deformation smoothness: We assume that the organs' motion evolves smoothly during image acquisition process therefore we expect the deformation on each DW-MR image to not deviate much from the deformation computed on each immediate neighbor, meaning the image preceding it. In the same time, each deformation field should deform the tissues in a way in which the anatomical information is retained. Therefore in order to impose temporal and spatial smoothness on the deformation fields we penalize the magnitude of displacement vector differences. The pairwise potential would then be:

$$
V\left(l_{p}^{d_{i}}, l_{q}^{d_{j}}\right)=\frac{\left\|\mathbf{d}^{\mathbf{l}_{\mathbf{p}}^{\mathbf{d}_{\mathbf{i}}}}-\mathbf{d}^{\mathbf{l}_{\mathbf{q}}^{\mathbf{d}_{\mathbf{j}}}}\right\|}{\|p-q\|}
$$

In case of temporal deformation smoothness $\left(V_{\text {inter }}\right) p \equiv q$ and $j=i+1$, whereas in case of spatial deformation smoothness $\left(V_{i n t r a^{1}}\right) i \equiv j$. For both cases we are assuming a 8-nodes connectivity.

Spatial smoothness on appearance model: Finally the last two terms in equation (14) relate to the spatial constraints applied within each of the images of our appearance model $\left(\mathbf{z}\right.$ and $\left.\mathbf{y}_{\mathbf{0}}\right)$. The squared error between two different appearance labels was used towards this end:

$$
\begin{aligned}
& V_{\text {intra }^{2}}\left(l_{p}^{z}, l_{q}^{z}\right)=\left(\mathbf{z}_{\mathbf{p}}^{\mathbf{z}}-\mathbf{z}_{\mathbf{q}}^{\mathbf{l}_{\mathbf{q}}^{\mathbf{z}}}\right)^{\mathbf{2}}, \\
& V_{\text {intra }^{3}}\left(l_{p}^{y_{0}}, l_{q}^{y_{0}}\right)=\left(\mathbf{y}_{\mathbf{0}}^{\mathbf{l}_{\mathbf{p}}^{\mathbf{l}}}-\mathbf{y}_{\mathbf{0}}^{\mathbf{l}_{\mathbf{q}}^{\mathbf{y}_{\mathbf{0}}}}\right)^{\mathbf{2}}
\end{aligned}
$$

Again we are assuming a 8-nodes intra-image connectivity.

\subsection{Optimization and implementation details}

Our discrete high-order MRF-based energy minimization problem was solved using Dual Decomposition (DD), which has been introduced as a framework for MRF optimization, offering global guarantees [11]. Dual Decomposition consists in decomposing a global difficult problem into smaller solvable subproblems (referred to as slaves) and then extracting a solution by combining the solutions from these subproblems. In our framework, the graph is decomposed into subgraphs that constitute the set of subproblems, such that they cover (at least once) every node and edge in each graph. One slave problem is defined for each line parallel to a coordinate axis in space and time (corresponding to pairwise interactions), and one slave is defined for the higher order clique (related to the data term). Two different optimization schemes are used for the solution of the subproblems: linear programming and duality [12] for pairwise interactions and exhaustive search for the higher order clique. In the end, global optimization is achieved through DD, that ensures agreement among the solutions provided by all slaves.

Our algorithm follows a coarse-to-fine process that first estimates parameters at a coarse spatial image resolution level and then updates the solution to a finer resolution 

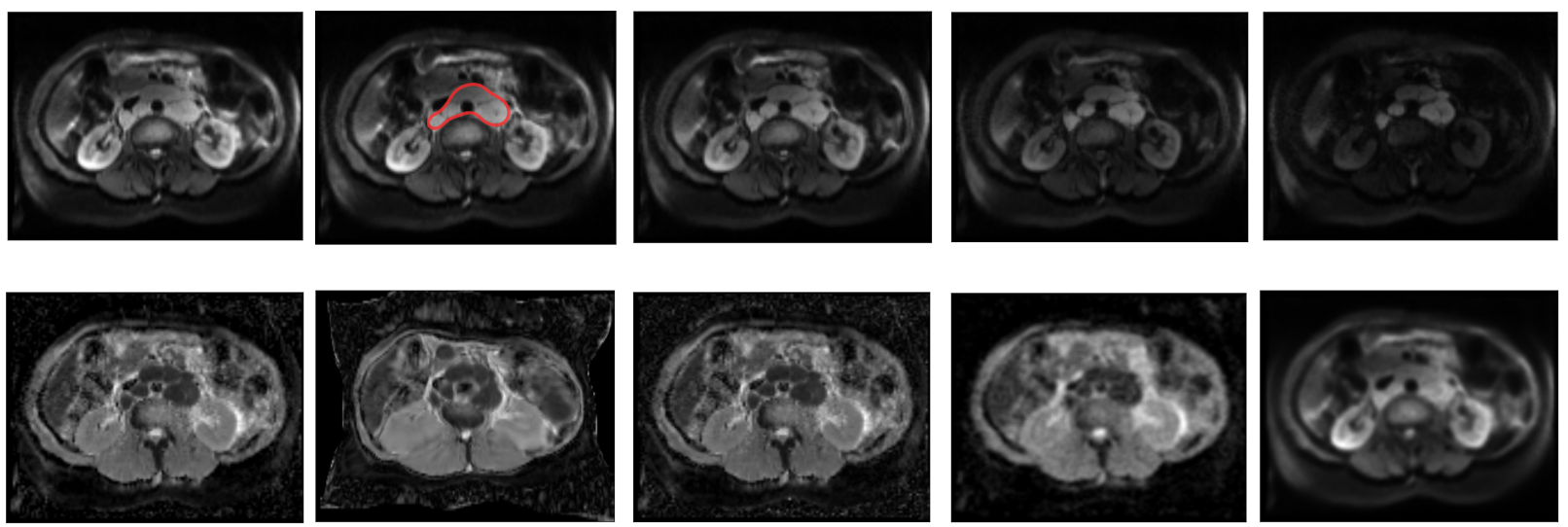

Figure 2: An axial slice of the 3D image of a patient. First row: $\mathrm{b}$ values of 20, 50, 150, 400 and $800 \mathrm{~s} / \mathrm{mm}^{2}$ (from left to right). The red contour in the "b 50" image corresponds to the tumor's contour, as it was drawn by the expert. 2nd row: ADC map derived by unregistered images, by deformed entropy-based group-wise registration approach, by curve fitting from the wraped by the proposed framework images and finally the ADC derived by the proposed joint approach (from left to right). The last image on the right is the $\mathbf{s}_{\mathbf{0}}$ derived also explicitly by our framework.

level. We applied a multi-resolution scheme with 3 resolutions levels; two levels in which the joint problem is solved and one at full image resolution in which only the appearance model is optimized, keeping the previously obtained deformation fields fixed. The rationale behind this multiresolution approach is that we want to adopt the grid size to the information content (frequencies) of the modeled variables, i.e. a sparse grid for the deformation model to cope with potential rigid transformations, a mid-level grid for local deformation and global intensity variations and a last dense grid for local intensity changes. Finally, regarding the run-time performance of the method, all the experiments were computed on a 8-cores machine $(3.6 \mathrm{GHz}$ processing power) with a memory requirement of $4 \mathrm{~GB}$.

\section{Experimental Validation}

In order to validate the proposed registration method, we used a data set provided by the Centre Hospitalier Universitaire Henri-Mondor, France. The data set consisted of 3D images of 17 different patients with lymphoma, scanned with a 3 Tesla MRI scanner using 5 b values: 20,50,150,400 and $800\left(\mathrm{~s} / \mathrm{mm}^{2}\right)$. Therefore, the sequence of the images to be registered was always of size $5(m=5)$ per patient. Both male and female patients were included. The image size was $130 \times 106 \times 22$ for every patient, with a pixel resolution of $2.46 \times 2.46 \mathrm{~mm}^{2}$ in the axial plane and $5 \mathrm{~mm}^{2}$ in the z-axis. Each image had been manually annotated by experts to indicate the contour of the tumor (see figure 2).
We compared the proposed joint method against three other approaches of computing an ADC map. The standard way of computing an ADC map is by plotting the logarithm of the relative signal intensity of the tissue on the $y$-axis against the $b$ values on the $x$-axis and then fitting a line through the plots with the use of linear regression (see equation (4)). Then the ADC is described by the slope of the line. This standard approach used in clinical practice does not take into account any motion correction scheme, therefore we refer to the ADC map derived by this approach as the unregistered case. We also compared the ADC derived by the proposed joint method against an ADC map computed based on deformed images, following the afore-mentioned ADC computation approach as a post-processing to the registration step. An implementation of a state-of-the art group-wise registration approach [24] was examined and the ADC computed based on the derived deformed images was calculated. An entropy estimator based on spacings [6] was used as a global similarity criterion and mutual information (MI) was used in the inter-images comparisons. Finally, we computed an ADC map which values were extracted from the wraped images by our approach. The ADC map in all cases except the case where it is computed explicitely by the proposed method was computed based on the DW-MR images (deformed or not). Then the accuracy on the ADC maps computed by these four different approached was quantitatively assessed by computing the sum-squared error (SSE) among the predicted image values derived by 
equation (1) and the actual (deformed or not) image values. (see Fig.1,right). The mean SSE over all voxels belonging to a tumor in the case of the explicitely computed by the proosed method ADC was lower than the corresponding mean SSE computed by all three rest cases.

Finally, in order to qualitatively evaluate the results, the ADC map for the same sagital view of a patient is shown in Fig.2a, as derived by the four different cases, as well as the $s_{0}$ image computed also explicitely by our method. Fig.2 (first row) illustrates an example of the DW-MR intensity variation for the five different $b$-valuess. In order to visually assess the results, we plot the ADC map of the same patient as calculated by four different methods. The results are illustrated in Fig. 2, second row, and correspond (from left to right) to the ADC calculated by voxel-wise curve fitting from (i) unregistered images, (ii) warped images using entropy-based similarity (implementation of the method in [24]), and (iii) warped images based on diffusion model fit (proposed approach without ADC and $\mathbf{y}_{0}$ regularization constraints). Finally the $\mathrm{AC}$ map an the $\mathbf{s}_{\mathbf{0}}$ image resuted from the propoed joint framework are illustated in the fourh and fith colum respectivly.

\section{Conclusion}

In this paper, we propose a novel joint deformable registration based on diffusion modeling that derives an optimal geometrical as well as appearance representation towards more accurately depicting the physiological process taking place during DW image acquisition. At that point we would like to note that convergence to a consenus solution among the slaves in the DD scheme was not achieved in all the experiments. In those cases, the majority voting algorithm was applied to derive the final solution to the energy minimization problem. Extensive study with more data and a parametric analysis of the method are required in order to better explore the predictive power of ADC. Overall, we conclude on the usefulness of DW-MRI registration towards improving the quality of ADC, which is in accordance with the conclusions derived by recent works on ADC refinement through image registration.

\section{Aknowledgements}

The authors of this paper would like to thank Enzo Ferrante and Maxim Berman for their helpful and constructive comments on the development of this work.

\section{References}

[1] D. Albano, C. Patti, L. La Grutta, F. Agnello, E. Grassedonio, A. Mulè, G. Cannizzaro, U. Ficola, R. Lagalla, M. Midiri, et al. Comparison between whole-body mri with diffusion-weighted imaging and pet/ct in staging newly diag- nosed fdg-avid lymphomas. European journal of radiology, 85(2):313-318, 2016.

[2] S. Alchatzidis, A. Sotiras, and N. Paragios. Local atlas selection for discrete multi-atlas segmentation. In International Symposium on Biomedical Imaging, 2015.

[3] A. Balbo-Mussetto, S. Cirillo, R. Bruna, A. Gueli, C. Saviolo, M. Petracchini, A. Fornari, C. Lario, D. Gottardi, A. De Crescenzo, et al. Whole-body mri with diffusionweighted imaging: a valuable alternative to contrastenhanced ct for initial staging of aggressive lymphoma. Clinical radiology, 2015.

[4] R. Bammer. Basic principles of diffusion-weighted imaging. European journal of radiology, 45(3):169-184, 2003.

[5] M. J. Black, D. J. Fleet, and Y. Yacoob. A framework for modeling appearance change in image sequences. In Computer Vision, 1998. Sixth International Conference on, pages 660-667. IEEE, 1998.

[6] S. Bouzebda, I. Elhattab, A. Keziou, and T. Lounis. New entropy estimator with an application to test of normality. Communications in Statistics-Theory and Methods, 42(12):22452270, 2013.

[7] B. Glocker, N. Komodakis, G. Tziritas, N. Navab, and N. Paragios. Dense image registration through mrfs and efficient linear programming. Medical image analysis, 12(6):731-741, 2008.

[8] J. Gu, T. Chan, J. Zhang, A. Y. Leung, Y.-L. Kwong, and P.-L. Khong. Whole-body diffusion-weighted imaging: the added value to whole-body mri at initial diagnosis of lymphoma. American Journal of Roentgenology, 197(3):W384W391, 2011.

[9] J.-M. Guyader, L. Bernardin, N. H. Douglas, D. H. Poot, W. J. Niessen, and S. Klein. Influence of image registration on apparent diffusion coefficient images computed from free-breathing diffusion mr images of the abdomen. Journal of Magnetic Resonance Imaging, 42(2):315-330, 2015.

[10] D.-M. Koh and D. J. Collins. Diffusion-weighted mri in the body: applications and challenges in oncology. American Journal of Roentgenology, 188(6):1622-1635, 2007.

[11] N. Komodakis, N. Paragios, and G. Tziritas. Mrf energy minimization and beyond via dual decomposition. Pattern Analysis and Machine Intelligence, IEEE Transactions on, 33(3):531-552, 2011.

[12] N. Komodakis, G. Tziritas, and N. Paragios. Performance vs computational efficiency for optimizing single and dynamic mrfs: Setting the state of the art with primal-dual strategies. Computer Vision and Image Understanding, 112(1):14-29, 2008.

[13] T. C. Kwee, T. Takahara, M. A. Vermoolen, M. B. Bierings, W. P. Mali, and R. A. Nievelstein. Whole-body diffusionweighted imaging for staging malignant lymphoma in children. Pediatric radiology, 40(10):1592-1602, 2010.

[14] D. Le Bihan, E. Breton, D. Lallemand, M. Aubin, J. Vignaud, and M. Laval-Jeantet. Separation of diffusion and perfusion in intravoxel incoherent motion $\mathrm{mr}$ imaging. Radiology, 168(2):497-505, 1988.

[15] C. Lin, A. Luciani, E. Itti, T. El-Gnaoui, A. Vignaud, P. Beaussart, S.-j. Lin, K. Belhadj, P. Brugières, E. Evan- 
gelista, et al. Whole-body diffusion-weighted magnetic resonance imaging with apparent diffusion coefficient mapping for staging patients with diffuse large b-cell lymphoma. $\mathrm{Eu}$ ropean radiology, 20(8):2027-2038, 2010.

[16] C. Lin, A. Luciani, E. Itti, C. Haioun, V. Safar, M. Meignan, et al. Whole-body diffusion magnetic resonance imaging in the assessment of lymphoma. Cancer Imaging, 12(2):403408, 2012.

[17] A. R. Padhani, G. Liu, D. Mu-Koh, T. L. Chenevert, H. C. Thoeny, T. Takahara, A. Dzik-Jurasz, B. D. Ross, M. Van Cauteren, D. Collins, et al. Diffusion-weighted magnetic resonance imaging as a cancer biomarker: consensus and recommendations. Neoplasia, 11(2):102-125, 2009.

[18] S. Parisot, H. Duffau, S. Chemouny, and N. Paragios. Joint tumor segmentation and dense deformable registration of brain $\mathrm{mr}$ images. In Medical Image Computing and Computer-Assisted Intervention-MICCAI 2012, pages 651658. Springer, 2012.

[19] S. C. Park, M. K. Park, and M. G. Kang. Super-resolution image reconstruction: a technical overview. Signal Processing Magazine, IEEE, 20(3):21-36, 2003.

[20] A. Pižurica and W. Philips. Estimating the probability of the presence of a signal of interest in multiresolution singleand multiband image denoising. Image Processing, IEEE Transactions on, 15(3):654-665, 2006.

[21] D. Rajan and S. Chaudhuri. Simultaneous estimation of super-resolved scene and depth map from low resolution defocused observations. Pattern Analysis and Machine Intelligence, IEEE Transactions on, 25(9):1102-1117, 2003.

[22] G. P. Schmidt, M. F. Reiser, and A. Baur-Melnyk. Wholebody mri for the staging and follow-up of patients with metastasis. European journal of radiology, 70(3):393-400, 2009.

[23] A. Sotiras, C. Davatzikos, and N. Paragios. Deformable medical image registration: A survey. Medical Imaging, IEEE Transactions on, 32(7):1153-1190, 2013.

[24] A. Sotiras, N. Komodakis, B. Glocker, J.-F. Deux, and N. Paragios. Graphical models and deformable diffeomorphic population registration using global and local metrics. In Medical Image Computing and Computer-Assisted Intervention-MICCAI 2009, pages 672-679. Springer, 2009.

[25] T. Takahara, Y. Imai, T. Yamashita, S. Yasuda, S. Nasu, and M. Van Cauteren. Diffusion weighted whole body imaging with background body signal suppression (dwibs): technical improvement using free breathing, stir and high resolution 3d display. Matrix, 160(160):160, 2004.

[26] H. Veeraraghavan, R. K. Do, D. L. Reidy, and J. O. Deasy. Simultaneous segmentation and iterative registration method for computing adc with reduced artifacts from dw-mri. Medical physics, 42(5):2249-2260, 2015.

[27] J. C. Vilanova and J. Barceló. Diffusion-weighted wholebody $\mathrm{mr}$ screening. European journal of radiology, 67(3):440-447, 2008.

[28] X. Wu, P.-L. Kellokumpu-Lehtinen, H. Pertovaara, P. Korkola, S. Soimakallio, H. Eskola, and P. Dastidar. Diffusionweighted mri in early chemotherapy response evaluation of patients with diffuse large b-cell lymphoma-a pi- lot study: comparison with 2-deoxy-2-fluoro-d-glucosepositron emission tomography/computed tomography. NMR in Biomedicine, 24(10):1181-1190, 2011.

[29] P. P. Wyatt and J. A. Noble. Map mrf joint segmentation and registration of medical images. Medical Image Analysis, 7(4):539-552, 2003.

[30] A. Yezzi, L. Zöllei, and T. Kapur. A variational framework for integrating segmentation and registration through active contours. Medical Image Analysis, 7(2):171-185, 2003. 